\title{
Liver transplantation for hepatocellular carcinoma: how far can the selection criteria be expanded?
}

\author{
Naglaa Allam
}

Received: 29 November 2011 / Accepted: 26 January 2012/Published online: 10 February 2012

(c) Asian Pacific Association for the Study of the Liver 2012

Hepatocellular carcinoma (HCC) is the fifth most common malignancy in the world and is associated with the third highest number of cancer-related deaths [1]. Surgical curative treatments for $\mathrm{HCC}$ include complete tumor resection and liver transplantation (LT). The LT offers the potential advantage of removing both the tumor and the organ at risk for developing future malignancies [2]. However, although LT is a widely accepted treatment for HCC, the basis for transplant selection can still be controversial.

The famous Milan criteria described in the landmark paper by Mazzaferro et al. [3] demonstrated that in patients with cirrhosis and a single tumor up to $5 \mathrm{~cm}$, or up to 3 lesions none larger than $3 \mathrm{~cm}$, and with no evidence of extrahepatic spread or macrovascular invasion, the 4-year actuarial and recurrence-free survival rates were 75 and $83 \%$, respectively. Milan criteria were rapidly established as the standard for transplantation and were introduced to clinical practice. Despite being validated by many studies, some believed that the Milan criteria were too restrictive and excluded a subset of patients with HCC who could have excellent outcomes if they were transplanted [4]. Yao et al. [5] further expanded the selection criteria and proposed the University of California at San Francisco (UCSF) criteria (a single tumor nodule with a diameter of up to $6.5 \mathrm{~cm}$ or 3 or fewer tumors, the largest of which has a diameter of $4.5 \mathrm{~cm}$ with the sum of the tumor diameters not more than $8 \mathrm{~cm}$, and without extrahepatic metastasis) on the basis of preoperative imaging findings with survival rates of $90 \%$ at 1 year and $75.2 \%$ at 5 years.

N. Allam $(\bowtie)$

Department of Hepatology and Liver Transplantation,

National Liver Institute, Shebin El kom, Egypt

e-mail: naglaaallam@yahoo.com
Currently, however, there is no international consensus regarding these approaches in clinical practice. Following the thought-provoking findings of UCSF's study, other groups have also ventured into pushing the limits set by the Milan criteria without compromising overall patient survival and HCC recurrence rate. Mazzaferro et al. [6] suggested the replacement of their strict Milan criteria with the use of the "up-to-seven" criteria, where the seven refers to the sum of the largest tumor in centimeters and the number of tumor nodules. In their retrospective survey of over 1,000 patients, the authors showed that a group of patients who had survival rates similar to those transplanted within the Milan criteria could be identified by the use of this "upto-seven" rule.

It is unlikely that the size and number of tumors reflect more than a marker for tumor biology and it is widely accepted that they are poor surrogates for the likelihood of the transplant "curing" the patient, but serological, molecular, or histological biomarkers are not yet sufficiently established to form a robust basis for treatment decisions [7]. Hence the challenge is to decide which histopathologic features, other than size and number, may better assess the biological behavior of HCC in these transplant recipients and add to the prognostic value. Microvascular invasion, defined as microscopic tumor invasion in smaller intrahepatic vessels, was stressed as a poor prognostic factor of liver resection (LR) and LT in several studies [8-10]; however, this was not clear in other reports $[5,11]$. It would be useful to clarify the significance of microvascular invasion for lesions within the "up-to-7 criteria." The study by Chan et al., reported in this issue of Hepatology International, investigated how significant microvascular invasion is compromising the long-term survival of LT recipients, regardless of whether a deceased donor graft or a living donor graft was used and also 
explored the survival benefits of primary LT over LR for patients with resectable HCC with or without microvascular invasion. The authors reported that the superiority of the 5-year disease-free survival rate of LT recipients versus LR patients was most remarkable in the presence of microvascular invasion. They found practically no difference between the overall and disease-free survival rates of LT recipients with HCC within the "up-to-seven" criteria, irrespective of whether the patients had vascular invasion. In their conclusion, primary LT for HCC with microvascular invasion and within the "up-to-seven" criteria doubled the chance of cure as compared with LR.

Further this study proposes expansion of the criteria for transplantation for HCC. From the technical standpoint, few aspects should be considered. This study is retrospective and is limited by the small number of the patients in each category. Only 15 patients were within the "up-toseven" criteria with microvascular invasion, which is not enough to provide strong significant results especially when the results are contradictory with the series of Mazzaferro where the 5-year survival of those with vascular invasion was decreased to $47 \%$. Chan et al. did not clearly define the degree of differentiation in those 15 patients with microvascular invasion. Although, tumor grade correlates with microvascular invasion, nevertheless, it could still be observed in patients with well-differentiated tumors $(25 \%)$. Hence, is application of this "up-to-seven criteria with microvascular invasion" limited to well-differentiated tumors?

The encouraging results in this extended criteria study by Chan et al. stimulate confirmation in future studies. Further pooling or accumulation of data from multiple centers will empower the study to draw a stronger conclusion before these criteria can be widely recommended in practice. Furthermore, the underlying etiology in over $85 \%$ of the patients in this study was hepatitis B. It would be interesting to recruit patients from Western and Egyptian centers to help in clarifying whether the finding from this study would be applicable to regions with a higher percentage of patients with hepatitis C. It is difficult to determine microvascular invasion pretransplant. Chan et al. based their analysis on data from the explanted liver rather than on pretransplant imaging, but they demonstrated that positron emission tomography (PET) showed a good correlation between increased (18)F-FDG uptake and microvascular invasion. This predictive value of PET was also previously reported by Kornberg et al. [12]. Confirming this information will be crucial as, indeed, risk stratification using a predictive model for post-LT HCC recurrence based on pre-LT imaging will help to guide candidate selection using these expanded criteria.

During the last two decades, operative mortality and surgical outcome of LT for HCC has improved. Prevention of tumor recurrence remains one of the most challenging tasks in current hepatology. Studies continue to explore expanding the Milan criteria. If the results of Chan et al. are confirmed in prospective multicenter studies, the "upto-seven irrespective of microvascular invasion criteria" reported will be undoubtedly of use to the clinician. With livers being a scarce resource and several competing indications, this study is of interest as it helps to pinpoint those outside the Milan criteria who might benefit from liver transplant.

\section{References}

1. Parkin DM, Bray F, Ferlay J, et al. Estimating the world cancer burden: GLOBOCAN 2000. Int J Cancer 2001;94:153-156

2. Abrams P, Marsh JW. Current approach to hepatocellular carcinoma. Surg Clin N Am 2010;90:803-816

3. Mazzaferro V, Regalia E, Doci R, et al. Liver transplantation for the treatment of small hepatocellular carcinomas in patients with cirrhosis. N Engl J Med 1996;334:693-699

4. Silva MF, Sherman M. Criteria for liver transplantation for HCC: what should the limits be? J Hepatol 2011;55(5):1137-1147

5. Yao FY, Ferrell L, Bass NM, et al. Liver transplantation for hepatocellular carcinoma: expansion of the tumor size limits does not adversely impact survival. Hepatology 2001;33:1394-1403

6. Mazzaferro V, Llovet JM, Miceli R, et al. Predicting survival after liver transplantation in patients with hepatocellular carcinoma beyond the Milan criteria: a retrospective, exploratory analysis. Lancet Oncol 2009;10(1):35-43

7. Neuberger J. Liver allocation for patients with hepatocellular carcinoma. Liver Transpl 2010;16:249-251

8. Allam N, Khalaf H, Fagih M, et al. Liver transplant for hepatocellular carcinoma: experience in a Saudi population. Exp Clin Transplant 2008;6:14-24

9. Hemming AW, Cattral MS, Reed AI, et al. Liver transplantation for hepatocellular carcinoma. Ann Surg 2001;233(5):652-659

10. Iwatsuki S, Dvorchik I, Marsh JW, et al. Liver transplantation for hepatocellular carcinoma: a proposal of a prognostic scoring system. J Am Coll Surg 2000;191(4):389-394

11. Zavaglia C, De Carlis L, Alberti AB, et al. Predictors of longterm survival after liver transplantation for hepatocellular carcinoma. Am J Gastroenterol 2005;100:2708-2716

12. Kornberg A, Freesmeyer M, Bärthel E, et al. 18F-FDG-uptake of hepatocellular carcinoma on PET predicts microvascular tumor invasion in liver transplant patients. Am $\mathrm{J}$ Transplant 2009;9:592-600 